01

\title{
Уравновешенные концентраторы потока и их применение для снижения электромагнитных сил в магнитных системах
}

\author{
(C) Г.А. Шнеерсон, Д.А. Дегтев \\ Санкт-Петербургский политехнический университет Петра Великого, \\ 195251 Санкт-Петербург, Россия \\ e-mail: gashneerson@mail.ru
}

Поступило в Редакцию 24 октября 2018 г.

В окончательной редакции 24 октября 2018 г.

Принято к публикации 24 октября 2018 г.

\begin{abstract}
Концентраторы магнитного потока - тела с радиальными разрезами, внесенные в поле магнита, существенно влияют на величину и распределение электромагнитных сил в магнитной системе. В приближении идеальной проводимости, справедливом при резко выраженном скин-эффекте, рассчитаны поля магнитных систем с внесенными в них концентраторами потока. На примере модельных задач показана возможность полной или частичной компенсации сил, воздействующих на концентратор. Вместе с тем показано, что его размещение вблизи обмотки магнита может снизить воздействующие на нее силы. Таким образом, концентратор разгружает обмотку, сам будучи при этом полностью или частично уравновешен. В итоге резко снижаются требования к устройствам, обеспечивающим прочность магнитной системы.
\end{abstract}

DOI: 10.21883/JTF.2019.06.47626.374-18

\section{Введение}

Концентратор представляет собой проводящий цилиндр с радиальным разрезом, который располагается соосно с обмоткой соленоида. При условии резко выраженного скин-эффекта поле не проникает в толщу проводника. По этой причине конфигурация поля в магнитной системе с концентратором потока может быть отличной от исходного поля соленоида. Концентраторы магнитного потока были предложены в 40-50-х годах прошлого века и описаны во многих работах. Они находят применение при высокочастотном нагреве проводников [1], используются для формирования области усиленного поля в приосевой области магнита [2-4], для создания требуемого распределения индукции при магнитной штамповке [5]. Новым применением этого устройства может быть создание области повышенного магнитного давления, обеспечивающей удержание торцевой части соленоида [6]. Существенно, что в ряде случаев конфигурацию магнитной системы можно выбрать так, что силы, воздействующие на концентратор, полностью или в значительной степени уравновешены. Подобные системы могут найти применение в технологии сильных импульсных магнитных полей. В настоящей работе рассматриваются модели, демонстрирующие возможности таких применений, и приводятся решения соответствующих специфических задач теории электромагнитного поля.

\section{1. Уравновешенный концентратор потока, расположенный в торцевой зоне магнита}

В рамках модельной задачи рассмотрим систему, представленную на рис. 1. В ней концентратор пред- ставляет собой кольцо 1 с радиальным разрезом 2. Оно расположено вблизи торцевой части одновиткового магнита 3. Границами концентратора являются коническая поверхность $T_{1}$, цилиндрические поверхности $T_{1}^{\prime}$, $T_{2}^{\prime \prime}$ и плоскость $T_{2}$, отстоящая от торца на расстояние $h$. Кольцо ограничено радиусами $R_{i}, R_{e}$, а торцевая часть обмотки - радиусами $R_{1}, R_{2}$. Далее при оценочных расчетах используем условия $h \ll R_{i}, R_{e}-R_{i}$. На примере данной модельной задачи покажем возможность создания уравновешенного концентратора потока. Кольцо находится в состоянии равновесия, если равна нулю равнодействующая аксиальных сил, создаваемых магнитным давлением. Особенность поля этой системы состоит в том, что магнитный поток $\Phi_{0}$ расщепляется на потоки $\Phi_{1}$ и $\Phi_{2}$, огибающие концентратор. Используем приближение идеальной проводимости, что допустимо в импульсном и высокочастотном полях, когда толщина скин-слоя мала. Потоки $\Phi_{1}$ и $\Phi_{2}$ индуцируют противоположно направленные токи $i_{1}$ и $i_{2}$. Первый из них распределен по поверхностям $T_{1}, T_{1}^{\prime}, T_{1}^{\prime \prime}$, а второй по поверхности $T_{2}$. Сумма этих токов равна нулю. Ток перетекает с одной поверхности на другую по краям радиального разреза. (Распределение тока по границам разреза рассмотрено ниже.)

Для дальнейших вычислений удобно следующим образом записать выражения для тока $i_{1}$ на поверхностях $T_{1}$, $T_{1}^{\prime}, T_{1}^{\prime \prime}$ и для аксиальной силы $F_{1, z}$ формируемой магнитным давлением

$$
\begin{gathered}
i_{1}=\xi \frac{\Phi_{1}}{\mu_{0} \pi R_{i}} . \\
F_{1, z}=\eta \frac{\Phi_{1}^{2}}{\mu_{0} \pi R_{i}^{2}} .
\end{gathered}
$$

Эти формулы вытекают из условий подобия. Безразмерные коэффициенты $\xi$ и $\eta$ определяются конфигурацией 

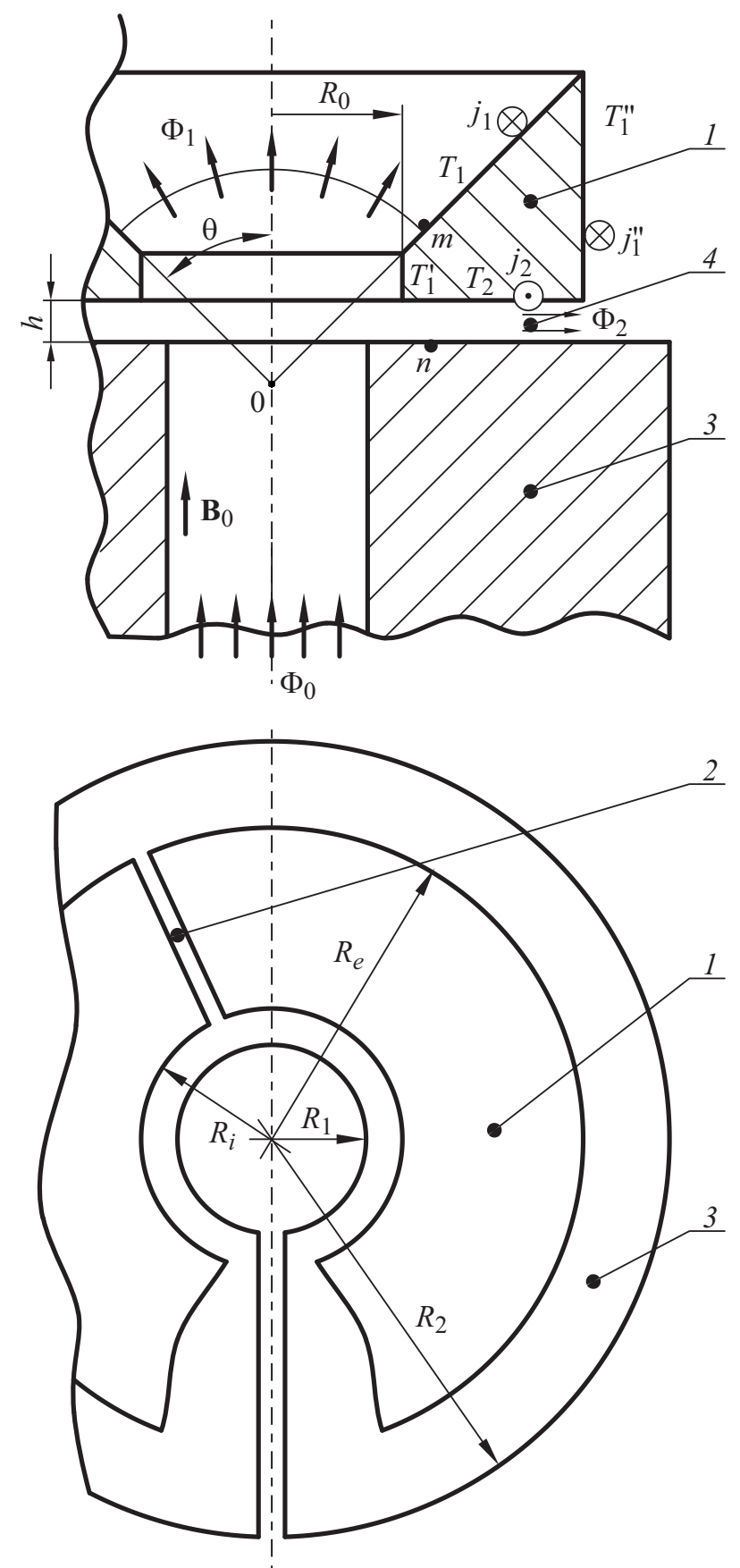

Рис. 1. Конический концентратор потока, расположенный вблизи торцевой границы одновиткового магнита: 1 - концентратор потока, 2 - радиальный разрез в теле концентратора, 3 - одновитковый магнит, 4 - зазор между концентратором и магнитом.

поверхностей $T_{1}, T_{1}^{\prime}, T_{1}^{\prime \prime}$ и отношениями характерных размеров магнитной системы $R_{e} / R_{i}, R_{1} / R_{e}, R_{2} / R_{1}, h / R_{1}$. При приближенном расчете тока на поверхности $T_{2}$ можно не учитывать поправку, обусловленную краевым эффектом у границы зазора в области с характерным размером порядка $h$. Это допустимо, если $h \ll R_{0}$, где $R_{0}$ - больший из радиусов $R_{i}, R_{1}$. В таком случае ток $i_{2}$ можно рассчитать по формуле

$$
i_{2}=-\int_{R_{0}}^{R_{e}} \frac{B(n)}{\mu_{0}} d r \approx-\int_{R_{0}}^{R_{r}} \frac{\Phi_{2} d r}{2 \mu_{0} \pi r h}=-\frac{\Phi_{2}}{2 \mu_{0} \pi h} \ln G,
$$

где $r$ - радиальная координата точки $n, G=R_{e} / R_{0}$. Из условия $i_{1}+i_{2}=0$ находим отношение потоков

$$
\frac{\Phi_{1}}{\Phi_{2}}=\frac{R_{i} \ln G}{2 h \xi} .
$$

Полученные формулы позволяют рассчитать индукцию в зазоре постоянной толщины $h$. Складывая потоки $\Phi_{1}$ и $\Phi_{2}$, получаем $\Phi_{1}+\Phi_{2}=\Phi_{0}$. В этой формуле $\Phi_{0}=\pi R_{1}^{2} B_{0}=\Phi_{2}\left(1+\Phi_{1} / \Phi_{2}\right)$ - поток в полости магнита вдали от его края, где индукция равна $B_{0}$. Отношение $\Phi_{1} / \Phi_{2}$ определяется формулой (2). Таким образом:

$$
\Phi_{1}=\Phi_{0} /(1+\delta), \quad \Phi_{2}=\delta \Phi_{0} /(1+\delta),
$$

где $\delta=2 h \xi /\left(R_{i} \ln G\right)$.

Поток в щели можно представить в виде $\Phi_{2} \approx 2 \pi R_{0} h B_{r}\left(R_{0}\right)$. Далее приходим к уравнению, связывающему характерные параметры магнитной системы:

$$
\frac{R_{i} R_{0}}{R_{1}^{2} \xi} \ln G+\frac{2 h R_{0}}{R_{1}^{2}}=\frac{B_{0}}{B_{r}\left(R_{0}\right)} .
$$

Число $\delta \ll 1$, если толщина зазора удовлетворяет условию $h \ll R_{0}, R_{e}-R_{0}$. Индукция в таком зазоре, рассчитанная без учета краевого эффекта, не зависит от толщины $h$ :

$$
B_{r}(r)=\frac{\Phi_{2}}{2 \pi r h} \approx \frac{\delta \Phi_{0}}{2 \pi r h}=\frac{\xi R_{1}^{2} B_{0}}{r R_{i} \ln G} .
$$

Можно найти аксиальную силу, воздействующую на поверхность $T_{2}$ концентратора:

$$
F_{2, z}=\int_{R_{0}}^{R_{e}} \frac{B^{2}(n)}{2 \mu_{0}} 2 \pi r d r \approx \frac{\Phi_{2}^{2}}{4 \mu_{0} \pi h^{2}} \ln G .
$$

Вследствие краевого эффекта в реальных системах ток $i_{2}$ и сила $F_{2, z}$ несколько отличаются от значений, представленных в формулах (1) и (4). Соответствующие поправочные множители могут быть найдены при численных расчетах.

Найдем отношение абсолютных значений аксиальных сил

$$
\lambda=\left|\frac{F_{1, z}}{F_{2, z}}\right|=\frac{4 \eta h^{2} \Phi_{1}^{2}}{R_{i}^{2} \ln G \Phi_{2}^{2}} .
$$

Используя формулу (2), получаем

$$
\lambda=\frac{\eta \ln G}{\xi^{2}}
$$

Характерно, что при условии $h \ll R_{0}, R_{e}-R_{0}$ отношение сил не зависит от значения зазора $h$. 
При равновесии концентратора имеет место равенство $F_{1, z}+F_{2, z}=0$ или $\lambda=1$. В этом случае отношение радиусов принимает значение $G_{0}$ определяемое формулой

$$
\ln G_{0}=\frac{\xi^{2}}{\eta} .
$$

Коэффициенты $\xi$ и $\eta$ определяются конфигурацией концентратора и зависят от отношения радиусов. Они могут быть найдены путем аналитического или численного расчета поля при различных $G$. Таким образом, выражение (5) - это уравнение, позволяющее найти отношение радиусов для уравновешенного концентратора. Сила, воздействующая на плоскость, вблизи которой расположен концентратор, определяется по формуле, вытекающей из равенств (4) и (3):

$$
F_{2, z}=\frac{\xi^{2} \pi R_{1}^{4} B_{0}^{2}}{\mu_{0} R_{i}^{2} \ln G(1+\delta)^{2}} .
$$

При равновесии концентратора она принимает вид

$$
F_{2, z}=\frac{\eta \pi R_{1}^{4} B_{0}^{2}}{\mu_{0} R_{i}^{2}(1+\delta)^{2}}
$$

Полученные выражения для тока $i_{1, z}$ и силы $F_{1, z}$ целесообразно сопоставить с оценками, выполненными в рамках модели источника потока $\Phi_{1}$, находящегося в точке 0 (рис. 1), и с результатами численных расчетов. Приведем оценки для концентратора, внешний радиус которого много больше внутреннего. В поле упомянутого источника в точке $m$ с координатой $r(m)>R_{0}$ изменение индукции описывается следующим образом:

$$
B(m)=\frac{\Phi_{1}}{\gamma \rho^{2}},
$$

где $\gamma=2 \pi(1-\cos \theta)=4 \pi \sin ^{2}(\theta / 2)-$ телесный угол, в котором распределен поток $\Phi_{1}, \rho=r(m) / \sin \theta$. Таким образом:

$$
B(m)=\frac{\Phi_{1} \cos ^{2}(\theta / 2)}{\pi r^{2}} .
$$

Ток на поверхности $T_{1}^{\prime \prime}$ много меньше, чем на $T_{1}$. Поэтому при вычислении тока $i_{1}$ можно ограничиться интегрированием по конической поверхности $T_{1}$ :

$$
i_{1} \approx \int_{R_{i}}^{R_{e}} \frac{B(m)}{\mu_{0}} \frac{d r}{\sin \theta} \approx \frac{\Omega \Phi_{1}}{2 \mu_{0} \pi} \operatorname{ctg}\left(\frac{\theta}{2}\right) \frac{1}{R_{i}} .
$$

Таким образом, в рассматриваемом примере $\xi=(\Omega / 2) \operatorname{ctg}(\theta / 2)$. Поправочный коэффициент $\Omega$ порядка единицы, зависящий от отношения $R_{e} / R_{i}=G$, учитывает отличие реальной конфигурации от указанной модели. Он определяется краевыми эффектами в окрестностях окружностей с радиусами $R_{i}$ и $R_{e}$ и током на цилиндрической поверхности $T_{1}^{\prime}$.
Далее можно найти аксиальную силу, воздействующую на поверхность $F_{1}$ концентратора:

$$
F_{1, z}=-\int_{R_{i}}^{R_{e}} \frac{B^{2}(m)}{2 \mu_{0}} 2 \pi r d r \approx \frac{-\Lambda \Phi_{1}^{2} \cos ^{4}(\theta / 2)}{2 \mu_{0} \pi} \frac{1}{R_{i}^{2}} .
$$

В данном примере $\eta=(\Lambda / 2) \cos ^{4}(\theta / 2)$, где $\Lambda-$ поправочный коэффициент. Условие равновесия (5) можно представить в виде

$$
\ln G_{0}=\frac{2 \Omega^{2}}{\Lambda \sin ^{2} \theta} .
$$

Коэффициенты $\Lambda$ и $\Omega$ зависят не только от угла $\theta$, но и от отношения $R_{e} / R_{i}$. Численные расчеты, выполненные методом конечных элементов с использованием программы Comsol Multiphysics, показывают, что в случае равенства внутренних радиусов кольца и магнита и при условиях $R_{2} \gg R_{e}, h=0.1 R_{i}$ у системы с углом $\theta=3 \pi / 8$ имеем $\Omega \approx 1.17$. $\Lambda \approx 2.14$. Коэффициенты $\lambda$ и $\Omega$ характеризуют роль краевого эффекта. Они показывают, что этот эффект слабо влияет на ток концентратора, но вносит большой вклад в расчетное значение аксиальной силы. Равновесие концентратора в этом примере имеет место при отношении радиусов $G_{0} \approx 3.85$.

\section{2. Концентратор в виде плоского кольца с разрезом}

Отношение радиусов для равновесного концентратора в виде тонкого плоского кольца $(\theta=\pi / 2)$ принимает меньшее значение, чем в приведенном выше примере. Например, для концентратора, отделенного от торца одновиткового магнита зазором $h=0.1 R_{1}$, имеющего внутренний радиус $R_{i}=R_{1}$ при условии $R_{2}>\mathrm{Re}$ равновесие имеет место, если $G_{0} \approx 2$. При этом $\Omega \approx 1$, $\Lambda \approx 2.23$.

Роль краевого эффекта существенно снижается у тонкого плоского концентратора с большим внутренним радиусом $\left(R_{i}>R_{1}, R_{2}>R_{e}\right)$. В такой системе коэффициенты $\Omega$ и $\Lambda$ близки к единице. При этом $\xi=0.5$, $\eta=0.125$. При указанных условиях отношение радиусов для плоского уравновешенного кольца малой толщины принимает значение $G_{0}=e^{2} \approx 7.39$.

Рассмотрим далее магнитную систему, в которой плоский концентратор толщиной $s$ и торцевая часть одновиткового магнита имеют одинаковые радиальные размеры $R_{1}=R_{i}, R_{2}=R_{e}$ (рис. 2 ). При условии $h \ll R_{1}$ распределения тока и магнитного давления по радиусу на внешней поверхности концентратора в этой системе являются практически такими же, как у одновиткового магнита. Данные, полученные путем численного расчета и приведенные на рис. 3 (кривая 1), показывают, что в диапазоне изменения отношений радиусов $2<G_{i}<5$ число $\lambda=\eta \ln G / \xi^{2}>1$. В отличие от рассмотренного 


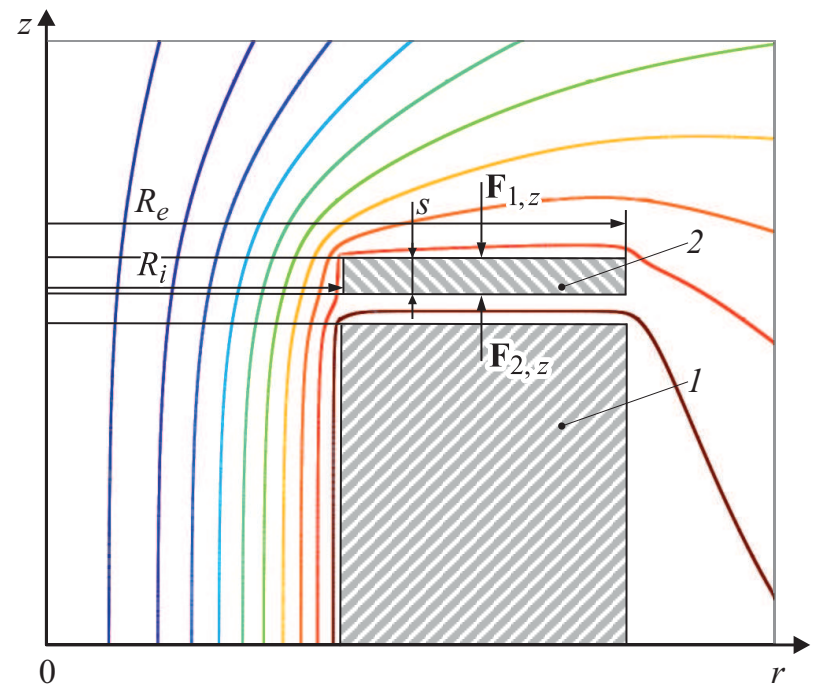

Рис. 2. К расчету сил, воздействующих на плоский концентратор потока, расположенный вблизи одновиткового магнита: 1 - магнит, 2 - плоский концентратор.

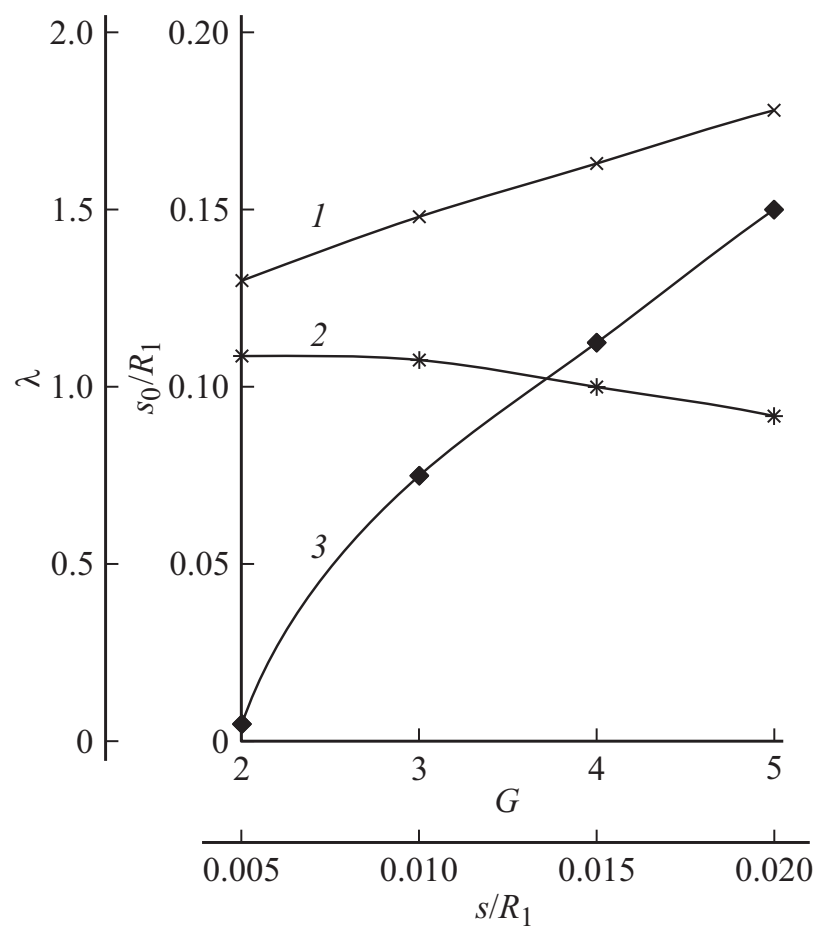

Рис. 3. Зависимости, характеризующие аксиальную силу, воздействующую на плоский концентратор: 1 - зависимость отношения аксиальных сил $\lambda=F_{1} / F_{2}$ от отношения радиусов для концентраторов малой толщины; $2-$ зависимость для магнитной системы с отношением радиусов $G=R_{2} / R_{1}=3$. 3 - зависимость $S_{0} / R_{1}=f(G)$ для уравновешенного концентратора.

выше примера равновесие кольца нулевой толщины при условии $R_{e}=R_{2}$ не имеет места, но оно может быть достигнуто у кольца конечной толщины $s$. При этом коэффициент $\eta$ изменяется мало, а коэффици- ент $\xi$ увеличивается. Это связано с тем, что при его расчете необходимо учитывать ток, индуцированный как на плоской, так и на цилиндрической частях поверхности отверстия кольца. Таким образом, условия равновесия плоского концентратора определяются не только отношением внешнего радиуса к внутреннему, но и отношением толщины кольца $s$ к радиусу $R_{i}$. Численные расчеты позволяют при заданном отношении радиусов $G$ построить зависимость $\lambda=f\left(s / R_{i}\right)$ и найти толщину концентратора $s_{0}$, соответствующую условию его равновесия $(\lambda=1)$. Примером может быть такая зависимость, построенная для магнитной системы с отношением радиусов $G=3$ (рис. 3 , кривая 2). В этом случае равновесие кольца имеет место, если его толщина принимает значение $s_{0} \approx 0.075 R_{i}$. Зависимость $s_{0} / R_{i}=f(G)$ представлена на рис. 3 (кривая 3).

Коэффициенты $\xi$ и $\eta$ определяются характером распределения тока по поверхности концентратора. Их значения могут варьировать не только путем изменения толщины кольца, но и путем изменения конфигурации границы. Это расширяет возможности построения уравновешенных концентраторов потока.

\section{3. Особенности распределения тока по краям разреза плоского концентратора магнитного потока}

Поверхностная плотность наведенного тока в общем случае по-разному распределена по радиусу на поверхностях $T_{1}+T_{1}^{\prime}+T_{1}^{\prime \prime}$ и $T_{2}$ (рис. 1). Перераспределение

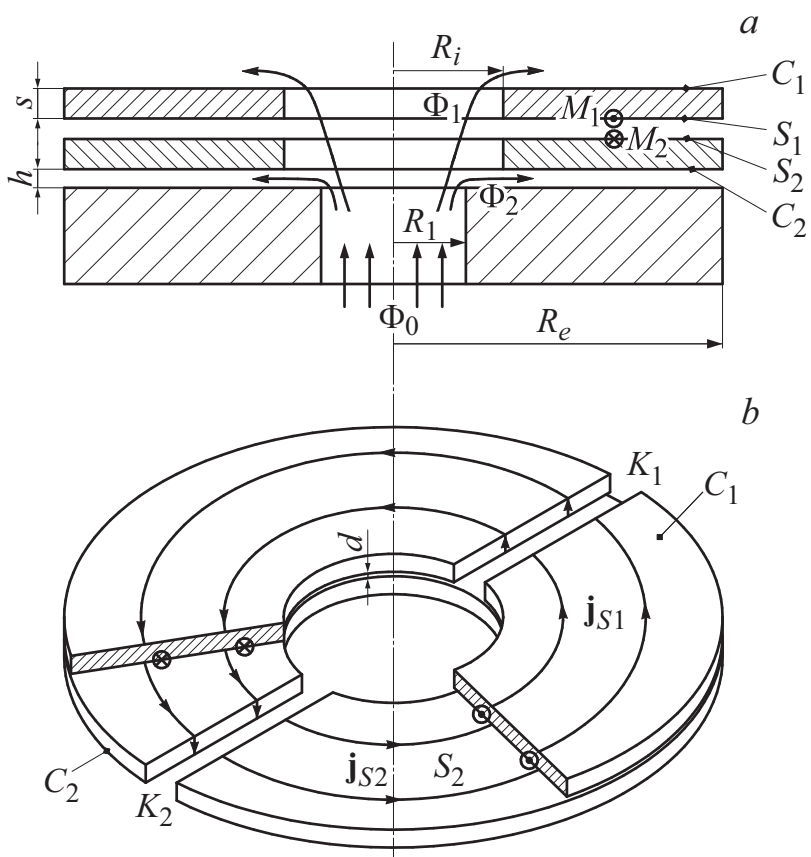

Pис. 4. $a-$ двуслойный концентратор, состоящий из дисков $C_{1}$ и $C_{2}$ - толщиной $s$, разделенных зазором $d ; b-$ распределение тока по поверхностям $S_{1}$ и $S_{2}$ дисков с разрезами $K_{1}$ и $K_{2}$. 
тока происходит в области радиального разреза 2 и в ее окрестности. Здесь нарушается аксиальная симметрия, и имеют место локальные особенности поля. В наименьшей степени это нарушение имеет место у концентратора, состоящего из двух колец с радиальными разрезами, разделенных зазором с малой толщиной $d$ (рис. $4, a, b)$. Кольца имеют противоположно расположенные разрезы $K_{1}$ и $K_{2}$ (рис. 4,b). Оба кольца с изоляционной прокладкой механически соединены и в совокупности могут рассматриваться как один плоский концентратор с внешними границами $C_{1}$ и $C_{2}$, где распределены наведенные азимутальные токи $j_{1}$ и $j_{2}$. Эти токи пересекают границы разрезов $K_{1}$ и $K_{2}$ и переходят на внутренние поверхности $S_{1}$ и $S_{2}$, где происходит перераспределение тока. Поле поверхностных токов $j_{S 1}$ и $j_{S 2}$ на границах зазора $d$, не обладает аксиальной симметрией, но эти токи удовлетворяют условию бифилярности [7]: $j_{S 1}\left(M_{1}\right)=-j_{S 2}\left(M_{2}\right)$, если точки $M_{1}$ и $M_{2}$ расположены на одном перпендикуляре к поверхностям $S_{1}$ и $S_{2}$ (рис. $4, a$ ). Это условие является следствием резко выраженного скин-эффекта. Оно может нарушаться лишь на малых участках с характерной шириной порядка $d$ вблизи границ поверхностей $S_{1}$ и $S_{2}$. Распределение тока по указанным поверхностям может быть найдено путем решения уравнения Лапласа для скалярного потенциала $U$, градиент которого связан с поверхностной плотностью тока соотношением [7]:

$$
\nabla U\left(M_{1}\right)=-\frac{\mu_{0} d}{2} j_{S 1}
$$

Это уравнение применимо на всей поверхности $S_{1}$, кроме области шириной порядка $d$ вблизи края. Радиальные разрезы $K_{1}$ и $K_{2}$ делят каждое из колец, ограниченных поверхностями $S_{1}$ и $S_{2}$, на два полукольца, в которых распределение тока одинаково. Оно определяется указанным решением уравнения Лапласа со следующими приближенными граничными условиями второго рода

$$
\begin{gathered}
\left.\frac{\partial U}{\partial \varphi}\right|_{r=R_{i, e}}=0,\left.\quad \frac{\partial U}{\partial \varphi}\right|_{K 1}=-j_{S 1} \frac{2}{\mu_{0} d}, \\
\left.\frac{\partial U}{\partial \varphi}\right|_{K 2}=-j_{S 2} \frac{2}{\mu_{0} d} .
\end{gathered}
$$

Первое из этих условий является следствием того, что поверхностный ток перетекает с внешних поверхностей на внутренние только через края разрезов, а не через края колец - окружности с радиусами $R_{i}$ и $R_{e}$. Два других условия описывают токи, переходящие с внешних поверхностей колец на внутренние через края разрезов.

Концентратор, показанный на рис. 4, $a$, расположен вблизи торцевой части одновиткового магнита. Для примера рассмотрим случай, когда выполнены условия $h, s \ll R_{i}, R_{i} \geq(2-3) R_{1}$ и $R_{e} \gg R_{i}$. При таких условиях краевые эффекты вблизи границ с радиусами $R_{i}$ и $R_{e}$ выражены слабо. Это позволяет принять, что распределение радиальной компоненты индукции и поверхностной плотности тока на поверхностях $S_{1}$ и $S_{2}$ в приближении идеальной проводимости описываются следующими формулами:

$$
\left(B_{r}\right)_{S 1}=\mu_{0} j_{S 1} \approx \frac{\Phi_{1}}{2 \pi r^{2}}, \quad\left(B_{r}\right)_{S 2}=-\mu_{0} j_{S 2} \approx \frac{\Phi_{2}}{2 \pi r h},
$$

где $\Phi_{1}$ - поток через круг радиуса $R_{i}, \Phi_{2}$ - поток в зазоре между концентратором и магнитом. Далее находим токи на наружных поверхностях колец:

$$
\begin{gathered}
i_{1}=\int_{R_{i}}^{R_{e}} j_{F 1} d r=\frac{\Phi_{1}(1-1 / G)}{2 \mu_{0} \pi R_{i}}, \\
i_{2}=\int_{R_{i}}^{R_{e}} j_{F 2} d r=\frac{-\Phi_{2}}{2 \mu_{0} \pi h} \ln G .
\end{gathered}
$$

Воспользовавшись условиями $i_{1}+i_{2}=0 \quad$ и $\Phi_{1}+\Phi_{2}=\Phi_{0}$, где $\Phi_{0}-$ поток в полости магнита, можно рассчитать радиальное распределение тока и на краях разрезов

$$
\begin{gathered}
j_{S 1}=j_{0} \frac{R_{i}^{2}}{r^{2}(1-1 / G)} \ln G, \\
j_{S 2}=-j_{0} \frac{R_{i}}{r},
\end{gathered}
$$

где

$$
j_{0}=\frac{\Phi_{0}}{2 \pi \mu_{0} R_{i} \ln G} \frac{(1-1 / G)}{h(1-1 / G)+R_{i} \ln G} .
$$

Решение уравнения Лапласа с граничными условиями (6) методом конечных элементов получено с помощью программы Comsol Multiphysics. Результат расчета для частного случая $R_{e} / R_{i}=3$ представлен на рис. 5 . Распределение тока по радиусу изменяется при переходе от зависимости вида (7) вблизи разреза $K_{1}$ на верхнем кольце к зависимости (8) вблизи разреза $K_{2}$ на нижнем.

При построении поля системы с плоским концентратором его можно рассматривать как сплошное кольцо, хотя практически он может быть реализован по описанной схеме в виде пары колец с радиальными разрезами. Если оба кольца механически скреплены, то сила, возникающая под действием магнитного давления поля в зазоре, не передается на остальные элементы магнитной системы.

Двуслойная система, аналогичная рассмотренной, может быть использована и при других конфигурациях концентраторов потока. В частности, цилиндрический концентратор (разрезной экран) может состоять из двух соосных тонкостенных цилиндров с противоположно расположенными разрезами.

Далее рассмотрены примеры, показывающие возможности использования уравновешенных концентраторов магнитного потока для снижения аксиальных сил в магнитах. 

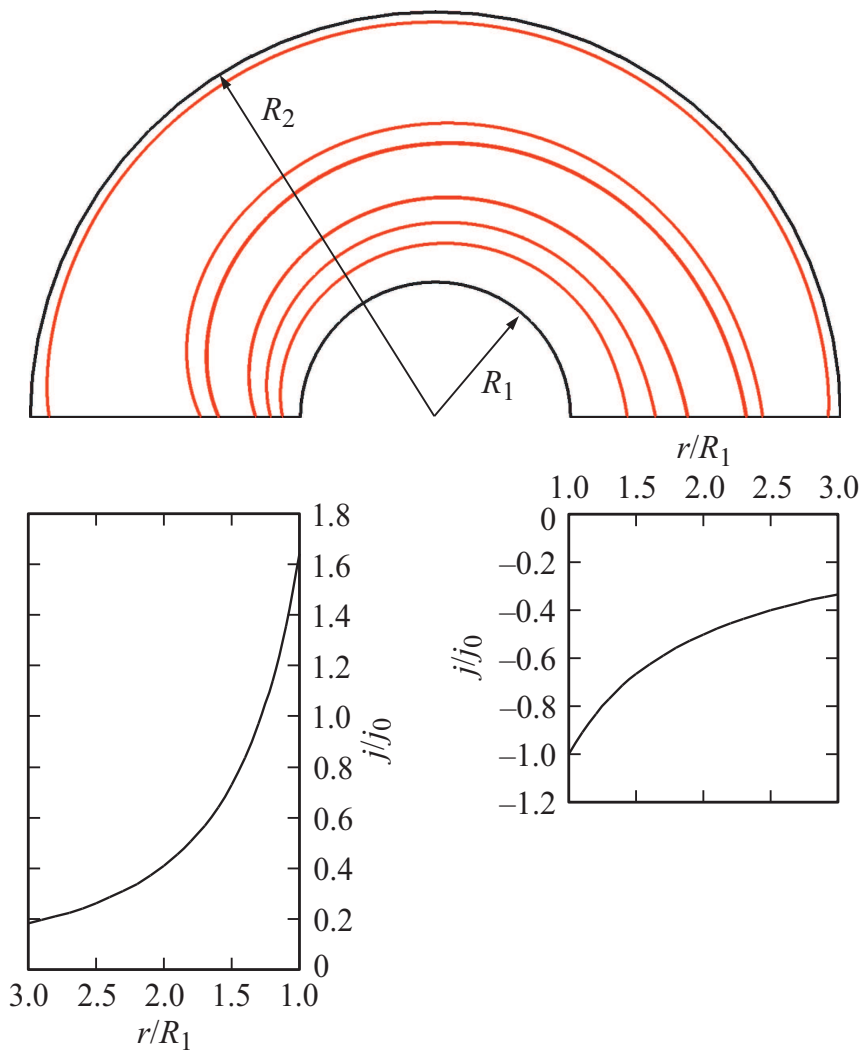

Рис. 5. Линии тока на поверхностях $S_{1}$ и $S_{2}$ и распределение тока по краям разрезов $K_{1}$ и $K_{2}$, расположенных на одном диаметре.

В общем случае магнитная система может иметь обмотку с заданным распределением тока и контура, на границе которых при резко выраженном скин-эффекте функция потока $\Psi$ принимает постоянное значение. На тех контурах, которые присоединены к источнику тока, эти значения заданы. Вместе с тем магнитная система может иметь несколько концентраторов потока. Значения функции потока на их границах $\Psi_{k}$ соответствуют условиям равенства нулю тока каждого концентратора:

$$
i_{k}=\sum_{n=1}^{N} c_{n k} \Psi_{n}+\Delta i_{k}=0 .
$$

В этих уравнениях $\Psi_{n}-$ функции потока на границах всех контуров магнитной системы, включая концентраторы, $\Delta i_{k}$ - значения тока, наведенного на границе $k$-го контура в поле обмотки с заданным током при условии равенства нулю значений функции потока всех контуров. Коэффициенты $c_{n k}$ в этом уравнении могут быть найдены путем дополнительных расчетов поля магнитной системы. Число таких расчетов равно числу контуров $N$. В каждом из этих расчетов на всех контурах принимается значение наведенного тока $\Delta i_{k}^{\prime}=0$ на контуре с порядковым номером $n$ задается граничное условие $\Psi_{n}^{\prime}=1$ а на остальных контурах функция потока принимается равной нулю. Каждый расчет позволяет найти токи $i_{k}^{\prime}$ на всех контурах с номерами $k$ и найти столбец матричных коэффициентов. Эти коэффициенты $c_{n k}$ численно равны токам $i_{k}^{\prime}$. Ниже приведен пример такого расчета для системы с двумя цилиндрическими концентраторами потока.

\section{4. Снижение аксиальной силы в многовитковой обмотке с помощью цилиндрических концентраторов потока}

Аксиальная сила $F_{0}$, воздействующая с одного конца на обмотку многовиткового магнита, определяется пересекающим ее магнитным потоком $\Phi_{\perp}$. В длинном соленоиде с обмоткой малой толщины и радиусом $R_{1}$
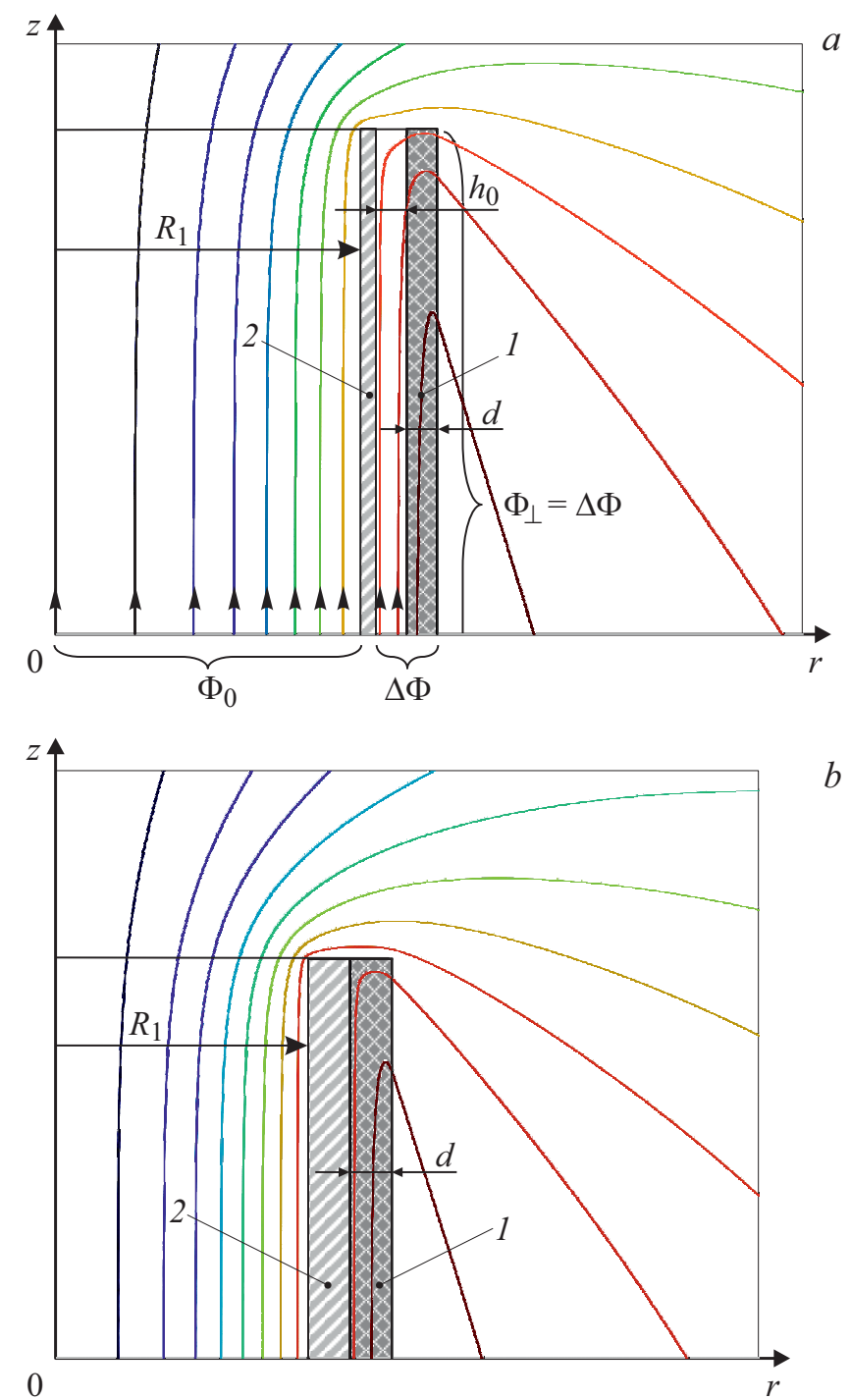

Рис. 6. Многовитковый магнит с цилиндрическим концентратором потока: $a-$ тонкостенная обмотка 1 с цилиндрическим концентратором (разрезным экраном) $2 ; b-$ пример магнита с тонкой обмоткой, примыкающей к экрану. 


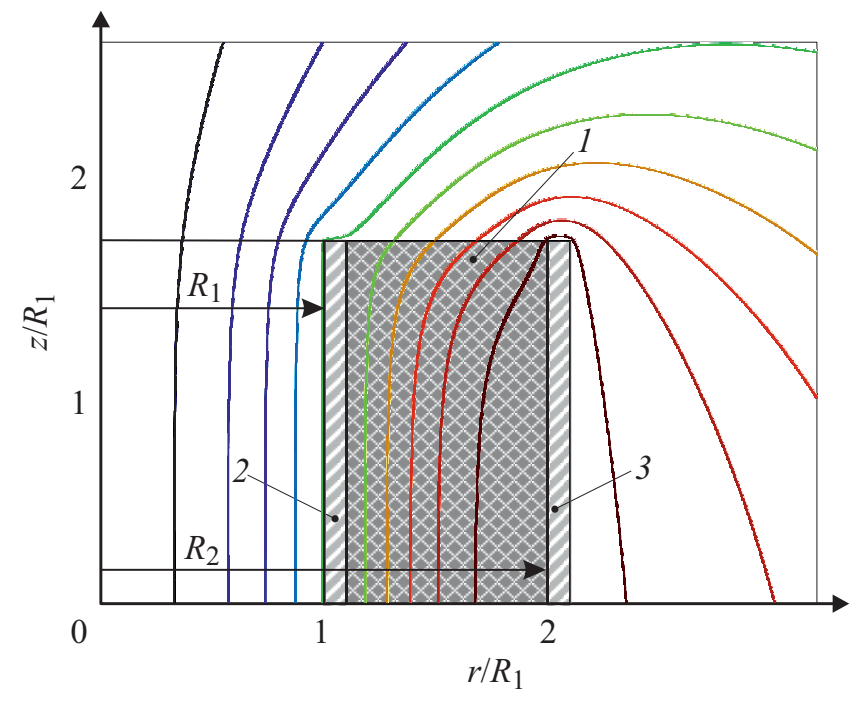

Рис. 7. Магнит с двумя разрезными экранами.

указанный поток есть $\Phi_{0} / 2$, где $\Phi_{0}=\pi R_{1}^{2} B_{0}$ - поток в полости магнита вдали от торцов, в области однородного поля с индукцией $B_{0}$. В таком соленоиде аксиальная сила может быть рассчитана по формуле [8,9]:

$$
F_{0}=-B_{0} \Phi_{\perp} / \mu_{0}=-\frac{\pi R_{1}^{2} B_{0}^{2}}{2 \mu_{0}} .
$$

В магнитной системе с цилиндрическим концентратором потока (разрезным экраном) эта сила может быть существенно уменьшена, поскольку в такой системе обмотку пересекает лишь поток $\Phi_{\perp}$, проходящий в зазоре $h_{0}$ межу экраном и обмоткой и в обмотке толщиной $d$ (рис. 6,a). При условии $h_{0} \ll R_{1}$ в магните большой длины индукции в полости магнита и в зазоре мало отличаются и близки к $B_{0}$, а поток в зазоре есть $\Delta \Phi=\Phi_{\perp} \approx 2 \pi R_{1} h_{0} B_{0}$. Если при этом толщина обмотки много меньше, чем зазор $h_{0}$, то аксиальная сила принимает значение

$$
F_{z} \approx-\frac{B_{0} \Delta \Phi}{\mu_{0}}=-\frac{2 \pi h_{0} R_{1} B_{0}^{2}}{\mu_{0}} .
$$

Следовательно, $F_{z} / F_{0} \approx 4 h_{0} / R_{1}$.

Существенное уменьшение аксиальной силы имеет место и в том случае, когда толщина обмотки относительно мала, но превышает толщину зазора $h_{0}$. Пример магнитной системы, в которой отношение толщины обмотки к ее внутреннему радиусу есть $d / R_{1}=0.15$, а $h_{0}=0$ приведен на рис. $6, b$. Численный расчет показывает, что в данном примере при равенстве толщины обмотки и концентратора отношение сил составляет $F_{z} / F_{0} \approx 0.21$. Таким образом, применение концентратора позволяет снизить аксиальную силу, если зазор между обмоткой и экраном и толщина обмотки малы по сравнению с радиусом магнита. В этой системе на экран действует аксиальная сила $F_{1, z} \approx 0.33 F_{0}$ и радиальное магнитное давление. Оно достигает наибольшего значения на краю, но быстро спадает при удалении от края: на расстоянии от него, равном радиусу $0.5 R_{1}$, разность магнитных давлений поля внутри и снаружи экрана составляет около $0.1 B_{0}^{2} / 2 \mu_{0}$. Таким образом, в длинном магните граница разрезного экрана уравновешена в радиальном направлении на большей части за исключением небольшого участка вблизи края.

В соленоиде с обмоткой большой толщины применение одиночного тонкостенного концентратора, размещенного внутри обмотки, дает менее заметный эффект. Это происходит из-за того, что большой поперечный поток формируется в самой обмотке. Существенно снижение этой силы может дать применение нескольких тонкостенных экранов. В представленном на рис. 7 примере магнитной системы с отношением внешнего радиуса обмотки к внутреннему $R_{2} / R_{1}=2$ используются два экрана толщиной $0.1 R_{1}$. Один из них прилегает к внутренней, а второй к внешней границе обмотки. Применение второго экрана приводит к существенному снижению поперечного потока. В такой системе к обмотке приложена сила $F_{z} \approx 0.28 F_{0}$. Аксиальные силы, действующие на внутренний и внешний экраны, в этом примере составляют $0.13 F_{0}$ и $0.2 F_{0}$, соответственно.

Рассмотренные примеры показывают, что цилиндрические концентраторы, обладающие достаточной прочностью, чтобы выдержать указанные осевые и радиальные нагрузки, позволяют существенно уменьшить осевую силу в многовитковом магните.

\section{5. Оценка возможности использования уравновешенного концентратора потока с плоской границей для удержания торцевой части магнита с квазибессиловой обмоткой}

Одной из актуальных проблем электрофизики является создание неразрушаемых магнитов для получения поля с индукцией мегагауссного уровня. В используемых в настоящее время магнитных системах с равнонагруженными обмотками достигнуто поле с индукцией, близкой к 100 Т. Из-за ограничений, обусловленных прочностью магнита, дальнейший рост индукции возможен при резком росте размеров магнитной системы. У таких магнитов отношение внешнего радиуса к внутреннему оценивается зависимостью

$$
G=R_{2} / R_{1} \approx \exp \left(B^{2} / 2 \mu_{0} \sigma_{M}\right),
$$

где $B-$ индукция генерируемого поля, $\sigma_{M}$ максимально допустимое механическое напряжение материала обмотки. Для получения поля с индукцией 150 Т при использовании сверхпрочного материала с $\sigma_{M}=2 \cdot 10^{9}$ Ра приведенная оценка дает $G \approx \exp 4.5 \approx 90$. Альтернативой традиционному методу получения поля в магнитах с азимутальным током может быть использование квазибессиловых магнитов. При всех трудностях их реализации 


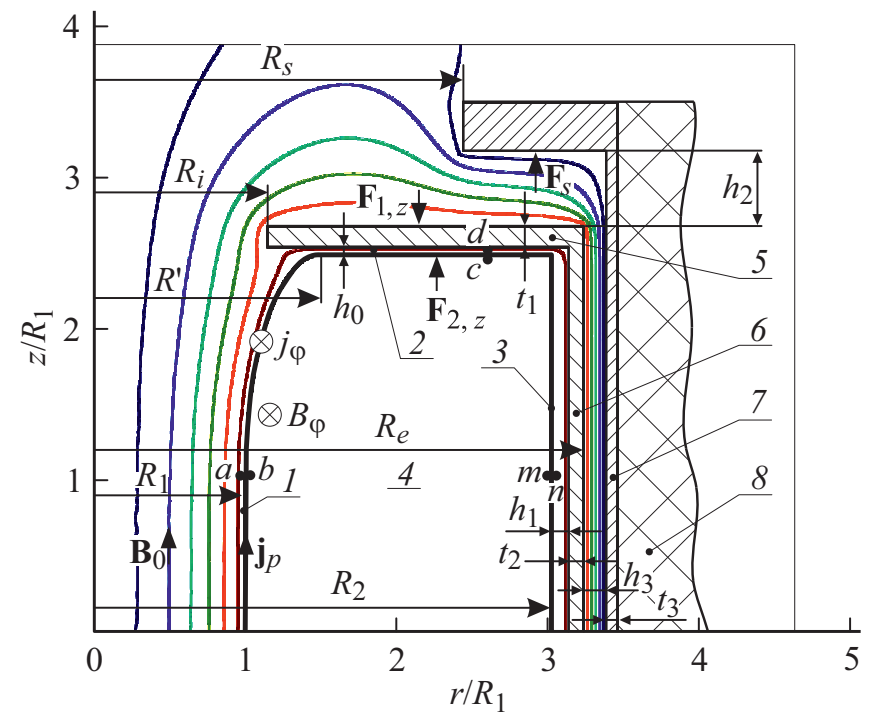

Рис. 8. Концентратор с плоской и цилиндрической частями и диамагнитный экран в магнитной системе соленоида с квазибессиловой обмоткой.

развитие этой технологии открывает перспективу достижения мегагауссных полей в неразрушаемых магнитах с обмоткой, выполненной из доступных материалов.

На рис. 8 показана схема одной из возможных конфигураций магнита с тонкой квазибессиловой обмоткой. Соленоиды с такими обмотками описаны в ряде работ, обзор которых содержится в монографии [9]. Обмотка магнита состоит из цилиндрической части 1 с внутренним радиусом $R_{1}$, плоской торцевой части 2 и внешней части 3 , ограниченной радиусом $R_{2}$. В рамках идеализированной базовой модели предполагается, что толщина квазибессиловой обмотки мала, а распределение в ней тока непрерывно. Ранее отмечалось, что в концентратоpax потока линейная плотность индуцированного тока имеет только азимутальную компоненту $j_{\varphi}$. В отличие от этого в квазибессиловой обмотке вектор линейной плотности тока $\mathbf{j}$ имеет как азимутальную компоненту $j_{\varphi}$, так и полоидальную $j_{p}$. При этом вектор $\mathbf{j}$ направлен под углом к оси симметрии, близким к $\pi / 4$. Индукция близкого к однородному аксиального поля в полости магнита есть $B_{0}$. В обмотке должно быть выполнено условие локального равновесия. Оно имеет место при равенстве магнитных давлений в точках $a$ и $b$, расположенных на одной нормали по разные стороны обмотки. В такой обмотке остаточные механические напряжения малы. Например, при непрерывном распределении тока напряжения, рассчитанные по формуле Мизеса в тонкой однослойной цилиндрической обмотке, близки к значению $\sigma_{M} \approx 0.16 B_{0}^{2} /\left(2 \mu_{0}\right)$, а в многослойной $-\sigma_{M} \approx 0.21 B_{0}^{2} /\left(2 \mu_{0} N^{2}\right)$ где $N$ - число слоев [11]. При отсутствии тока во внешней зоне 4 индукция азимутального поля в этой области есть

$$
B_{\varphi}(r)=B_{0} R_{1} / r
$$

Радиальный размер магнита выбирается так, чтобы магнитное давление на внешней границе $P_{m}=\left(B_{0}^{2} / 2 \mu_{0} G^{2}\right)$ не превышало значения, определяемого прочностью устройств, воспринимающих это давление. В примере с индукцией $B_{0}=150$ Т магнитное давление $P_{m}$ на внешней границе при отношениях $G=3$ и 4 составляет $10^{9}$ и $0.57 \cdot 10^{9} \mathrm{~Pa}$ соответственно. Эти оценки показывают, что в отличие от магнитов традиционного исполнения в рассмотренной системе приемлемые нагрузки могут иметь место без чрезмерного увеличения отношения радиусов. Однако для реализации магнита с квазибессиловой обмоткой должно быть обеспечено равновесие обмотки не только в цилиндрической, но и в торцевой части. В этой зоне должно быть выполнено условие локального равновесия обмотки: равенств магнитных давлений в точках $d$ и $c$. Это условие можно обеспечить, если использовать концентратор потока, состоящий из плоского кольца 5 толщиной $t_{1}$ и цилиндрической части 6 толщиной $t_{2}$. В зазоре постоянной толщины $h_{0}$ между концентратором 5 и торцевой частью обмотки 2 индукция должна изменяться по такому же закону, как и азимутальное поле во внешней зоне магнита. При этом по обе стороны торцевой части обмотки равны модули касательной компоненты индукции азимутального $B_{\varphi}$ (в точках $c$ ) и полоидального поля $B_{r}$ (в точках $d$ ). Вместе с тем обмотка должна быть поверхностью постоянного потока полоидального поля. В таком случае равна нулю нормальная к границе компонента индукции и отсутствует азимутальная компонента силы. Обмотка, удовлетворяющая указанным условиям, должна иметь „скругленный“ переходной участок, ограниченный радиусом $R^{\prime}$. Конфигурация этого участка может быть рассчитана методом итераций, как это описано в [11] и других работах [10]. В системе без концентратора условие равновесия и отсутствия нормальной компоненты индукции может быть выполнено на участке $R_{1}<r<1.64 R_{1}$. В рассматриваемом примере (рис. 8) граница „скругленного“ участка построена путем вариаций ее формы на участке с ограниченным изменением радиуса: $R^{\prime}=1.5 R$. При расчетах принято условие $h_{0} / R_{1}=0.05_{1}$. В данной системе может быть использован уравновешенный плоский концентратор. Равновесие концентратора, в присутствии которого в зазоре создается поле с требуемым значением индукции, удается обеспечить с помощью короткозамкнутого цилиндра 7 - диамагнитного экрана с внутренним радиусом $R_{S}$, отделенного зазором $h_{2}$ от плоской и $h_{3}-$ от цилиндрической части концентратора. В данной системе уравновешены плоская и цилиндрическая части концентратора 6. Равновесие внешней части обмотки 3 (равенство абсолютных значений индукции в точках $m$ и $n$ ) обеспечивается путем выбора зазора $h_{1}$ между обмоткой и цилиндрической частью концентратора 6. Индукция азимутального поля на внешнем крае квазибессиловой обмотки (в точке $m$ с радиальной координатой $\left.r(m)=R_{2}\right)$ есть $B_{0} R_{1} / R_{2}=B_{0} / G$. Указанный зазор выбирается таким, чтобы цилиндр 6 был уравновешен в радиальном направлении. Это имеет место, если индукция полоидального поля в зазорах $h_{1}$ и $h_{3}$ близка к индукции азимутального поля $B_{\varphi}\left(R_{2}\right)=B_{0} R_{1} / R_{2}$. При таких условиях магнитное давление, действующее на 
экран 7, близко к значению $P_{m}\left(R_{2}\right)=\left(1 / G^{2}\right) B_{0}^{2} / 2 \mu_{0}$. Удержание цилиндрической части экрана может быть обеспечено с помощью толстостенного внешнего бандажа 8 , напряжения в котором близки к $P_{m}\left(R_{2}\right)$. Для сравнения отметим, что в равнонагруженной обмотке обычного исполнения с азимутальным током механическое напряжение близко к $(1 / \ln G) B_{0}^{2} / 2 \mu_{0}[10]$. У магнита с отношением $G=R_{2} / R_{1}=3$ это значение примерно в 8 раз выше приведенной оценки $P_{m}\left(R_{2}\right)$.

В представленном примере принято указанное отношение радиусов и путем пробных расчетов выбраны размеры $R_{i}=1.15 R_{1}, R_{S}=2.5 R_{1}, h_{1}=0.03 R_{1}, h_{2}=0.3 R_{1}$, $t_{2}=0.14 R_{1}, t_{3}=0.13 R_{1}$. При этом аксиальная сила $F_{2, z} \approx 1.58 \pi R_{1}^{2} B_{0}^{2} / 2 \mu_{0}$. Расположение и размеры элементов магнитной системы подбираются так, что выполняется как условие равенства нулю полного тока концентратора, так и условие его равновесия в аксиальном направлении $\left|F_{1, z} / F_{2, z}\right| \approx 1$. Экран подвержен воздействию относительно небольшой аксиальной силы $F_{S}$. Эта сила в рассмотренном примере составляет около $0.36 F_{2, z}$. В работе [10] показана возможность использования плоского диамагнитного экрана для обеспечения равновесия торцевой части обмотки в импульсном магнитном поле. В такой системе аксиальная сила существенно больше: $F_{S}=F_{2, z}$. Чтобы избежать смещения экрана за время импульса, предлагается использовать его инерционное удержание с помощью груза большой массы. Применение концентратора потока позволяет примерно в три раза снизить аксиальную нагрузку на диамагнитный экран и существенно облегчить создание системы удержания торцевой части магнита. Таким образом, применение уравновешенного концентратора позволяет не только обеспечить условие локального равновесия обмотки, но и резко снизить нагрузку на систему ее удержания.

\section{Заключение}

Взаимодействие магнитного поля с током, наведенным на поверхности концентратора потока, создает встречно направленные аксиальные силы. При определенных условиях имеет место компенсация этих сил, и концентраторы могут находиться в равновесии. Найдены условия равновесия концентратора, имеющего форму кольца с радиальным разрезом, расположенного вблизи плоского торца одновиткового магнита. Эти условия слабо зависят от толщины зазора между концентратором и магнитом и выполняются при определенных соотношениях радиальных размеров и толщины кольца. Соответствующие зависимости рассчитаны для плоского кольца с такими же внутренним и внешним радиусами, как у магнита. На примере концентратора, состоящего из двух плоских колец с разрезами, разделенных тонким зазором, рассмотрено распределение тока, перетекающего с одной на другую поверхность концентратора. Концентратор в виде тонкого цилиндра с разрезом может быть использован для уменьшения аксиальной силы, воздействующей на обмотку тонкостенного многовиткового магнита. В случае обмотки большой толщины целесообразно использовать два соосных цилиндра, расположенных в толще обмотки. Использование концентраторов потока в сочетании с короткозамкнутым диамагнитным экраном позволяет обеспечить локальное равновесие торцевой части квазибессилового магнита. При этом конфигурация магнитной системы может быть построена так, что сумма аксиальных сил, воздействующих на концентратор, равна нулю, а аксиальная сила, воздействующая на экран в присутствии концентратора, много меньше, чем в системе без концентратора. Таким образом, применение концентратора дает возможность создания магнитов с квазибессиловой обмоткой и развитой торцевой частью. В таком магните нагрузка на систему удержания обмотки внешним бандажом резко снижена по сравнению с равнонагруженными магнитами обычного исполнения.

\section{Финансирование работы}

Работа поддержана грантом Российского Научного Фонда № 18-19-00230.

\section{Список литературы}

[1] Kim Y.B., Platner E.D. // Rev. Sci. Istr. 1959. Vol. 30. N 7. P. 524-533.

[2] Wilson M.N., Srivastava K.D. // Rev. Sci. Istr. 1965. Vol. 36. N 8. P. $1096-1100$.

[3] Карасик В.Р. Физика и техника сильных магнитных полей. М.: Наука, 1964.

[4] Ditz H., Lippman H.-J., Schenk H. // ETZ-A. 1967. Vol. 88. P. 475-480.

[5] Белый И.В., Фертик С.М., Хименко Л.Т. Справочник по магнитно-импульсной обработке металлов. Харьков: Вища школа, 1977. $168 \mathrm{c}$.

[6] Shneerson G.A., Danilin K.A., Neneshev A.P., Parfentev A.A. et al. // IEEE Transaction on Plasma Sci. 2017. Vol. 45. N 11. P. 3038-3041.

[7] Шнеерсон Г.А. Поля и переходные процессы в аппаратуре сверхсильных токов. 2-е изд., переработанное и дополненное. М.: Энергоатомиздат, 1992. 413 с. [Shneerson G.A. Fields and Transients in Superhigh Pulse Current Devices. NY: Nova Science Publishers, Inc. 1997. P. 561.]

[8] Askenasy S. // Physica B. 1992. Vol. 177. P. 36-40.

[9] Shneerson G.A., Dolotenko M.I., Krivosheev S.I. Strong and Superstrong Pulsed Magnetic Fields Generation. Berlin: De Gruyter, 2014. 429 c.

[10] Shneerson G.A., Koltunov O.S., Shneider-Muntau H.J., Titkov V.V., Parfentjev A.A. // Physica B. 2004. Vol. 346-347. P. 566-570.

[11] Шишигин С.Л. Электричество. 2008. № 9. С. 51-57. 